\title{
Smartphones, Babys, Kleinkinder und Kinder im Jahr 2021
}

\author{
Korrespondenzadresse \\ Prof. Dr. Dr. Manfred Spitzer \\ Universität Ulm \\ Leimgrubenweg 12-14 \\ 87054 Ulm, Deutschland
}

Bibliografie

Nervenheilkunde 2021; 40: 848-852

DOI 10.1055/a-1500-2520

ISSN 0722-1541

(C) 2021. Thieme. All rights reserved.

Georg Thieme Verlag KG, Rüdigerstraße 14,

70469 Stuttgart, Germany
Smartphones wurden nicht zum Gebrauch für Babys, Kleinkinder und Kinder entwickelt. Es handelt sich vielmehr um Werkzeuge mit vielfältigen Nutzungsmöglichkeiten für berufstätige Erwachsene: Dem Handwerker ersetzt es das Büro, die Dokumentation und bietet zugleich den Zugang zu technischen Spezifikationen jeglicher Materialien, Produkte, Apparaturen und Ersatzteile. Erreichbarkeit (über Kurznachrichten, E-Mail oder Telefon), weltweite Kommunikation in Wort und Bild, Zugang zu Daten und verschiedene Eingabemöglichkeiten für Daten sowie eine ausgeklügelte Auswahl von Antennen und Sensoren ermöglichen Funktionalitäten über Hunderttausende von Anwendungen (Apps), von denen man noch vor wenigen Jahren nicht einmal geträumt hätte: Um welchen Flug handelt es sich bei dem Flugzeug gerade über mir? Und hat er Verspätung? Welcher/welche/welches Stern/Pflanze/Insekt/(setzen Sie selbst hier irgendetwas ein) ist das? Man „filmt“ das zu bestimmende Objekt einfach und erhält die Antwort nach einigen Sekunden. Das geht auch schon mit manchen medizinischen Diagnosen (Hautkrebs), QR-Codes füllen langsam, aber sicher unser visuelles Umfeld, um nicht mehr von UNS, sondern von unserem Smartphone erkannt zu werden, was mit irgendeiner Funktionalität verbunden ist (bin ich geimpft? habe ich Corona?).

\section{Viel Zeit mit YouTube, arme Kinder}

In den westlichen entwickelten Ländern spielen mehr als 90\% der Kinder bereits im Kleinkindalter mit Smartphones und Tablets. Die mit digitalen Bildschirmmedien insgesamt verbrachte Zeit steigt von 49 Minuten bei den unter 2-Jährigen auf 2 Stunden und 30 Minuten bei den 2- bis 4-Jährigen und auf 3 Stunden und 5 Minuten bei den 5- bis 8-Jährigen an ( $\triangleright$ Abb. 1). 73\% der Zeit wird mit dem Betrachten von Videos verbracht, weitere $16 \%$ mit Computerspielen, die restlichen $11 \%$ entfallen auf Lesen (3\%), Hausaufgaben ( $1 \%$ ) oder andere nicht weiter genannte Tätigkeiten. Jungs schauen im Durchschnitt 35 Minuten länger als Mädchen, jeweils die Hälfte dieser Zeit wird mit Videos und Computerspielen zugebracht [17].

Interessant ist auch an dieser relativ neuen Erhebung, dass Online-Video (YouTube) und Streaming-Dienste (Netflix) das traditionelle Fernsehen abgelöst haben und DVDs kaum noch eine Rolle spielen ( $\triangleright$ Abb. 2).

Betrachtet man den YouTube-Konsum von Kindern inhaltlich, so dominieren Werbung, Spielzeug und Spiele (Computer-Games), wie die Auswertung von 1639 Videos ergab [14]. Volle $95 \%$ der YouTube-Videos für Kinder enthalten Werbung, 45 \% der Videos, die Kinder unter 8 Jahren ansehen, enthalten Werbung für Produkte und $27 \%$ enthalten nicht altersgemäße Inhalte. $30 \%$ der Videos, die die Kinder in diesem Alter sehen, enthalten Gewalt. Eine weitere Studie fand in 50 Videos für Kinder insgesamt 286 Werbespots [22], die teilweise nicht altersgerecht waren. Von großer Bedeutung ist weiterhin, dass der Medienkonsum vor allem bei den Kindern aus einkommensschwachen Familien deutlich angestiegen ist ( $\triangleright$ Abb. 3). Die Eltern in diesen Familien geben wesentlich häufiger an, dass die Inhalte der Bildung ihrer Kinder nutzen und dass die Kinder von Medien profitieren würden. Lediglich im Hinblick auf die körperliche Aktivität sind sich die Eltern weitgehend einig darüber, dass Bildschirmmedien einen schädlichen Effekt auf die Kinder haben. Aber dennoch glauben immerhin $16 \%$ aller Eltern, dass Bildschirme sich positiv auf sportliche Aktivitäten auswirken.

Lag der Unterschied der Bildschirmmediennutzung zwischen der niedrigsten und der höchsten Einkommensgruppe im Jahr 2013

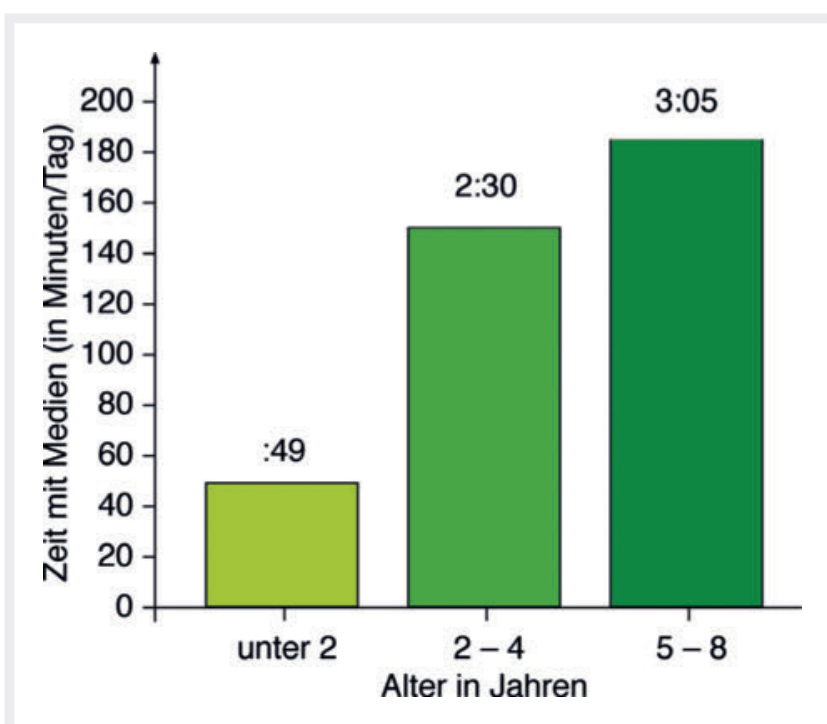

- Abb. 1 Durchschnittliche Zeit der Bildschirmmediennutzung von unter 2-Jährigen, 2- bis 4-Jährigen und 5- bis 8-Jährigen in den USA im Jahr 2020 (nach Daten aus [17]). 


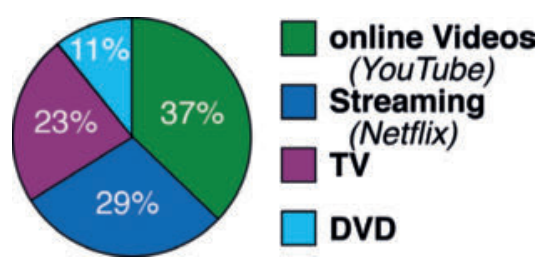

- Abb. 2 Prozentuale Verwendung unterschiedlicher Plattformen für Bewegtbilder durch Kinder im Alter zwischen 0 und 8 Jahren. Was früher „Film und Fernsehen“ war, sind heute YouTube und Netflix (nach Daten aus [17]).

noch bei 29 Minuten, war er im Jahr 2020 auf fast 2 Stunden angewachsen. Oder anders ausgedrückt: Die Bildschirmnutzung von Kindern in Haushalten mit niedrigem Einkommen hat seit 2013 um durchschnittlich 1:31 pro Tag zugenommen (von 2:17 auf 3:48 pro Tag), während er bei Kindern aus Haushalten mit höherem Einkommen praktisch gleichgeblieben ist (von 1:48 auf 1:52). Dieser zunehmende Unterschied bei der Bildschirmmediennutzung nach Einkommen ist vor allem auf die Ausweitung des Zugangs zu mobilen Geräten zurückzuführen, die von Familien mit niedrigem Einkommen viel begeisterter angenommen wurden als von einkommensstärkeren Familien ( $\triangleright$ Abb. 4).

„Als die Nutzung von Smartphones und Tablets im Jahr 2017 zuzunehmen begann, wuchs sie unter afroamerikanischen Kindern und Kindern aus einkommensschwachen Familien; dieser Trend war im Jahr 2020 noch deutlicher ausgeprägt, “ kommentieren die Autoren ihre Ergebnisse [17]. Dieser Trend ist nicht auf die USA beschränkt, sondern lässt sich nahezu überall feststellen. Vor allem in Asien ist die Nutzung von Smartphones häufig noch weiter verbreitet als hierzulande. In Afrika ist das Smartphone oft das erste und einzige digitale Endgerät überhaupt. Dort wurde nicht nur das Telefon-Festnetz übersprungen, sondern vielfach auch der PC oder Laptop. Obwohl Kinder sich besonders rasch an Smartphones und Tablets gewöhnen, wurden weder die Geräte noch die auf ihnen laufende Software für Kinder entwickelt. Schon gar nicht berücksichtigten die Entwickler die Tatsache, dass Kinder auf die Verstärkung von Verhaltensweisen besonders rasch und zugleich unreflektiert reagieren, also beispielsweise auf rasche Bildfolgen, leuchtende Farben, animierte (Comic-) Figuren, schnelle Bewegungen und automatisierte Funktionen wie Autoplay. Daher fällt es Kleinkindern besonders schwer, sich vom Smartphone oder Tablets zu lösen.

\section{Wutanfälle}

Was bedeutet diese Entwicklung für die Kinder? In einer Studie mit dem schönen englischen Titel „Tablets, toddlers and tantrums“ (zu Deutsch - leider ohne Stabreim - etwa: „Tablets, 2-Jährige und Wutanfälle“) ging die Arbeitsgruppe um die US-amerikanische Kinderärztin Jenny Radesky dieser Frage nach [7]. Sie untersuchten, ob Kleinkinder - im Vergleich zu einem gedruckten Buch - nach dem Spielen mit einem Tablet mehr Wutanfälle, eine geringere Kooperationsbereitschaft und Bereitschaft, Aufforderungen nachzukommen, zeigten. Es wurden 72 Eltern-Kleinkind-Paare in ein Wohnzimmerlabor eingeladen, um eine Videoaufzeichnung beim Spielen

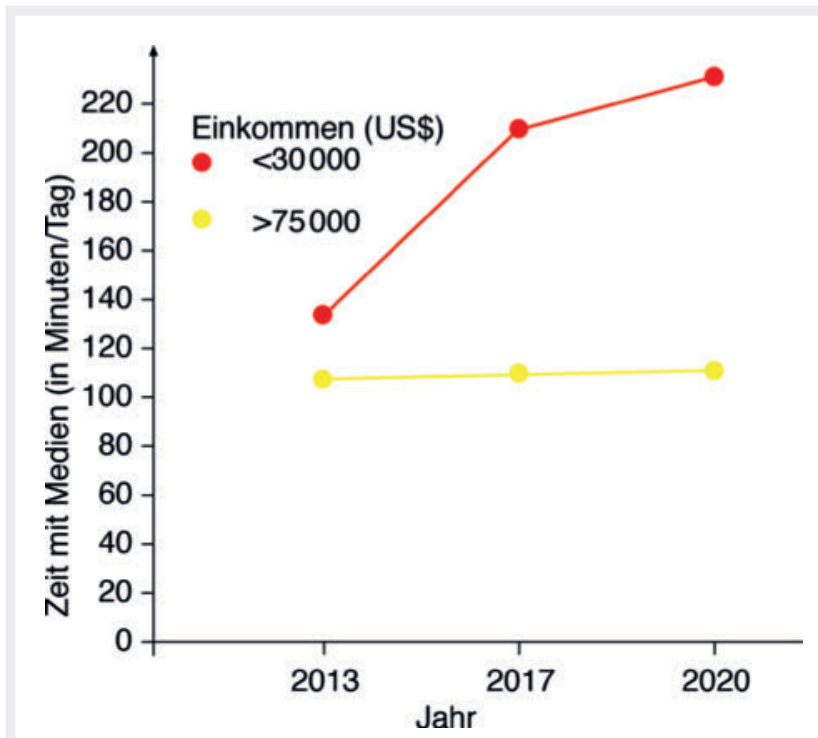

- Abb. 3 Durchschnittliche Zeit der Bildschirmmediennutzung von 0 - bis 8-Jährigen aus Familien mit niedrigem ( $<30000$ US\$/Jahr) und hohem Einkommen (>75000 US\$/Jahr) in den USA im Jahr 2020 (nach Daten aus [17]).

mit einem Tablet oder einem Buch vorzunehmen. Inhaltlich ging es um Kinderreime auf dem Tablet oder im Druckformat. Es gab insgesamt 3 Spielbedingungen, die in randomisierter Reihenfolge für jeweils 3 Minuten gespielt werden sollten:

- erweiterte Tablet-App (Bewegtbild, Ton und automatisches Abspielen),

- einfache Tablet-App (Bewegtbild, Ton und Soundeffekte beim Antippen von Hotspots),

- gedrucktes Buch (erstellt aus Screenshots der Tablet-App).

Die Tablet-Apps und das gedruckte Buch befanden sich in Schachteln mit der Bezeichnung 1-3, in einem Regal. Die Eltern-Kind-Paare wurden angewiesen, mit jedem Format (Tablet/Tablet/Print) jeweils 3 Minuten lang zu spielen und das Spielzeug dann auf die Schachtel zu legen. Die beiden Zeitabschnitte zwischen den 3 Spielepisoden wurden als „Übergänge“ 1 und 2 bezeichnet. In diesen Zeiträumen wurde das Verhalten der Kleinkinder aufgezeichnet und kodiert. Wenn nichts weiter geschah, wurde dies mit „0“ kodiert, gab es jedoch Weinen, verbale Beschwerde, Schreien, Frustration oder gar Wutanfälle (was reliabel festgestellt werden konnte; $r=0,88-0,96)$; wurde eine „1 “ kodiert. Beim ersten Übergang gab es einen klaren Unterschied zwischen Tablet und Buch: Nach dem Spielen mit dem Tablet gab es in $22 \%$ (Tablet, Bedingung 1) bzw. $25 \%$ der Fälle Verhaltensauffälligkeit, wohingegen es nach dem Spielen mit dem Buch keine (0\%) Wutanfälle und Ähnliches gab. Nach dem zweiten Übergang gab es numerisch (aber nicht signifikant) deutlich weniger Wutanfälle nach dem Buch als nach den beiden Tablets ( $\triangleright$ Abb. $\mathbf{5}$ ). Die Autoren gehen davon aus, dass Verhaltensauffälligkeiten bei Kleinkindern nach dem Spielen mit dem Tablet das Ergebnis von Designmerkmalen von Tablet-Programmen (Apps) sind, durch die die Kinder an das Tablet und seine Funktionen relativ stark gebunden werden. Wie auch in der Werbung, die bei Kindern mindestens so gut funktioniert wie bei Erwachsenen, 


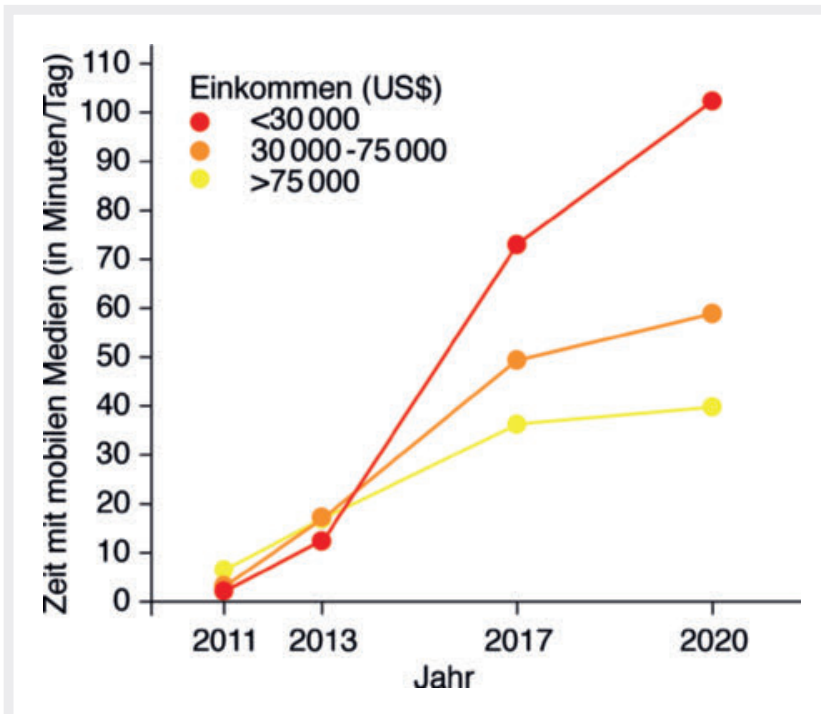

- Abb. 4 Durchschnittliche Zeit der Nutzung mobiler Endgeräte (Smartphone, Tablet) von 0- bis 8-Jährigen aus Familien mit niedrigem (<30 000 US\$/Jahr), mittlerem (30000 bis 75000 US\$/Jahr) und hohem Einkommen (>75000 US\$/Jahr) in den USA im Jahr 2020 (nach Daten aus [17]).

wird die Aufmerksamkeit der kleinen Kinder gleichsam usurpiert und nicht mehr losgelassen. Deswegen kommt es dann durch die abrupte Unterbrechung des Tablet-Spiels zu Wutausbrüchen.

Die Studie ist deswegen von besonderem Wert, weil sie gewissermaßen mit der Lupe untersucht hat, was genau passiert, wenn man Kindern Tablets und Smartphones gibt. Dies ist deswegen von großer Bedeutung, weil man diese Ergebnisse in einen größeren Rahmen stellen kann, der dann ihre Bedeutung verdeutlicht.

\section{Das Experiment mit dem versteinerten Gesicht}

Der US-amerikanische Entwicklungspsychologe Edward Tronick [20], Direktor der Abteilung für Entwicklungspsychologie und Professor an der Universität von Massachusetts, Boston, entwickelte schon vor Jahrzehnten zusammen mit dem Pädiater Berry Brazelton das sogenannte "Still-face-Experiment" zur Untersuchung von Neugeborenen und Kleinkindern. Dabei wird eine liebevolle Kontaktsituation von Angesicht zu Angesicht zwischen Mutter und Kind für etwa eine Minute unterbrochen. Die Mutter wendet sich ab und zeigt dem Kind dann ein versteinertes, ausdrucksloses Gesicht. Das Kind reagiert darauf in verschiedenen Phasen: Zunächst versucht es mit verschiedensten Methoden wieder die Aufmerksamkeit der Mutter zu bekommen, schließlich reagiert es mit körperlichem und emotionalem Rückzug. Diese Situation simuliert Interaktionen beispielsweise depressiver Mütter, von denen man weiß, dass sich ihr zurückgezogenes und wenig responsives Verhalten negativ auf die kindliche kognitive und emotionale Entwicklung auswirkt. Auch unsicher gebundene, nicht ausreichend feinfühlige Mütter können diese Auswirkungen auf die kindliche Entwicklung haben. Langfristig können immer wieder erfolgende derartige Abbrüche von erlebter Gemeinsamkeit zu Störungen im Sozialverhalten und anderen psychischen Störungen führen.

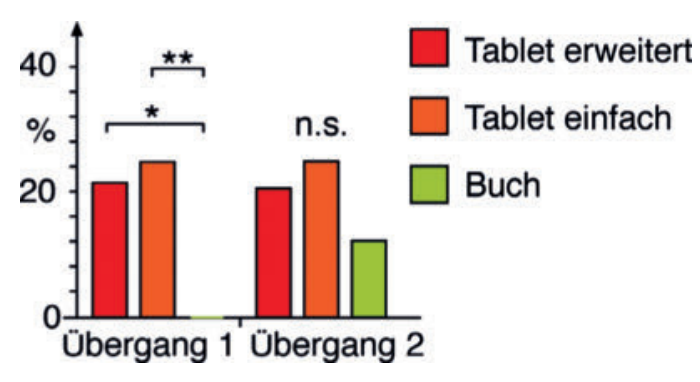

- Abb. 5 Prozentsatz der Verhaltensauffälligkeiten (Wutausbrüche) von Kleinkindern nach dem Spielen mit Tablets (und 2 unterschiedlichen Programmen) sowie nach dem Spielen mit einem Buch, wobei inhaltlich das Gleiche gespielt wurde $\left({ }^{*} \mathrm{p}<0,05 ;{ }^{* *} \mathrm{p}<0,01\right.$ nach Daten aus [7]).

Schaut die Mutter plötzlich auf ihr Smartphone, z. B. weil eine SMS eingetroffen ist, geschieht letztlich nichts anderes als ein Still-face-Experiment [4, 18], die Kinder heischen nach Aufmerksamkeit, verzweifeln zunehmend, schreien erregt und wenden sich am Ende frustriert ab. Solche Unterbrechungen der Eltern-Kind-Interaktion durch den Smartphone-Gebrauch sind mittlerweile wissenschaftlich untersucht und zeigen, dass durch die mütterliche Mediennutzung die Reaktionsfähigkeit und das pädagogische Verhalten während der Unterbrechungsphase abnahmen. Außerdem erhöhten die Kinder während der Unterbrechung ihre Aufmerksamkeitsgebote, was verdeutlicht, dass die Erkenntnisse zum Stillface-Experiment auf diese Situation übertragen werden können. Unterbrechungen durch Smartphones verringerten die Qualität der Eltern-Kind-Interaktionen und damit die Qualität der Elternschaft. Weitere Studien zeigten einen Zusammenhang zwischen der Nutzung des Smartphones zur Beruhigung kleiner Kinder und deren Verhaltensauffälligkeiten, die mit einer pädiatrischen Symptom-Checkliste erfasst wurden [8].

Halten wir fest: Sehr viele Menschen, insbesondere Eltern, die den Alltag mit Kindern bewältigen müssen, haben den Eindruck, dass ihnen das Smartphone die Arbeit nicht leichter macht. Gute Studien hierzu liegen jedoch kaum vor, denn Wissenschaft braucht Zeit. Sogar die langfristigen Folgen von übermäßigem Smartphone-Gebrauch sind mittlerweile publiziert. In einer Übersicht vom 29. Mai 2021 fassen israelische Wissenschaftler diese wie folgt zusammen: „Komorbidität mit Depressionen, Angstzuständen, Zwangsstörungen, ADHS und Alkoholproblemen; exzessive Smartphone-Nutzung wird mit Schwierigkeiten bei der Regulation von Emotionen und Aufmerksamkeit sowie mit Impulsivität, beeinträchtigten kognitiven Funktionen, Abhängigkeit von sozialen Netzwerken, Schüchternheit und geringem Selbstwertgefühl in Verbindung gebracht. In medizinischer Hinsicht sind Schlafprobleme, verminderte körperliche Fitness, ungesunde Essgewohnheiten, Schmerzen und Migräne, verminderte kognitive Kontrolle und Veränderungen des Volumens der grauen Substanz des Gehirns zu nennen “ [21]. Die Autoren enden mit einer klaren Schlussfolgerung: „Exzessive Smartphone-Nutzung ist mit psychiatrischen, kognitiven, emotionalen, medizinischen und Gehirnveränderungen verbunden, die von Gesundheits- und Bildungsexperten berücksichtigt werden sollten. " Neuseeländische Psychiater sprechen 
mittlerweile von einer digitalen Pandemie [1]. Seit dem 15. Januar 2021 ist in China der Gebrauch von Smartphones an allen Schulen zum Schutz der Gesundheit der Schüler verboten [25], und seit dem 1. September 2021 sind Online-Spiele für Personen unter 18 Jahren in China fast völlig verboten. Minderjährige dürfen nur noch an Freitagen, Samstagen sowie Sonn- und Feiertagen jeweils eine Stunde zwischen 20 und 21 Uhr am Computer spielen. Wiederum wurde das Verbot mit dem Schutz der körperlichen und geistigen Gesundheit Minderjähriger begründet. Wann wachen wir auf, nennen die Probleme beim Namen und beginnen damit, die nächste Generation vor den Folgen des Smartphones zu schützen?

Literatur

[1] Augner C, Vlasak T, Aichhorn W et al. Tackling the „digital pandemic": The effectiveness of psychosocial intervention strategies in problematic Internet and Smartphone use - A meta-analysis. Australian and New Zealand Journal of Psychiatry 2021; doi: $10.1177 / 00048674211042793$

[2] Domoff SE, Radesky JS, Harrison K et al. A Naturalistic Study of Child and Family Screen Media and Mobile Device Use. J Child Fam Stud 2019; 28 (2): 401-410. doi:10.1007/s10826-018-1275-1

[3] Konrad C, Berger-Hanke M, Hassel G et al. Does texting interrupt imitation learning in 19-month-old infants? Infant Behav Dev 2021; 62: 101513. doi:10.1016/j.infbeh.2020.101513

[4] Konrad C, Hillmann M, Rispler J et al. Quality of Mother-Child Interaction Before, During, and After Smartphone Use. Front Psychol 2021; 12: 616656. doi:10.3389/fpsyg.2021.616656

[5] McDaniel BT, Radesky JS. Longitudinal Associations Between Early Childhood Externalizing Behavior, Parenting Stress, and Child Media Use. Cyberpsychol Behav Soc Netw 2020; 23 (6): 384-391. doi:10.1089/cyber.2019.0478

[6] McDaniel BT, Radesky JS. Technoference: longitudinal associations between parent technology use, parenting stress, and child behavior problems. Pediatr Res 2018; 84 (2): 210-218. doi:10.1038/s41390018-0052-6

[7] Munzer TG, Miller AL, Wang Y et al. Tablets, toddlers and tantrums: The immediate effects of tablet device play. Acta Paediatr 2021; 110: 255-256

[8] Radesky JS, Carta J, Bair-Merritt M. The 30 Million-Word Gap: Relevance for Pediatrics. JAMA Pediatr 2016; 170 (9): 825-6. doi:10.1001/ jamapediatrics.2016.1486

[9] Radesky JS, Christakis DA. Increased Screen Time: Implications for Early Childhood Development and Behavior. Pediatr Clin North Am 2016; 63 (5): 827-39. doi:10.1016/j.pcl.2016.06.006

[10] Radesky JS, Christakis DA. Keeping Children's Attention: The Problem With Bells and Whistles. JAMA Pediatr 2016; 170 (2): 112-3. doi:10.1001/jamapediatrics.2015.3877
[11] Radesky JS, Kistin C, Eisenberg S et al. Parent Perspectives on Their Mobile Technology Use: The Excitement and Exhaustion of Parenting While Connected. J Dev Behav Pediatr 2016; 37 (9): 694-701. doi:10.1097/DBP.0000000000000357

[12] Radesky JS, Peacock-Chambers E, Zuckerman B et al. Use of Mobile Technology to Calm Upset Children: Associations With Social-Emotional Development. JAMA Pediatr 2016; 170 (4): 397-9. doi:10.1001/ jamapediatrics.2015.4260

[13] Radesky JS, Schaller A, Yeo SL et al. Young kids and YouTube: How ads, toys, and games dominate viewing. San Francisco, CA: Common Sense Media; 2020

[14] Radesky JS, Weeks HM, Ball R et al. Young Children's Use of Smartphones and Tablets. Pediatrics. 2020; 146 (1): e20193518. doi:10.1542/peds.2019-3518

[15] Radesky JS. Establishing Early Literacy Habits in a Profit-Driven Digital World. Pediatrics 2021; 147 (6): e2020047472. doi:10.1542/ peds.2020-047472

[16] Radesky JS. Young children's online-offline balance. Acta Paediatr 2021; 110 (3): 748-749. doi:10.1111/apa.15649

[17] Rideout V, Robb MB. The Common Sense census: Media use by kids age zero to eight. Common Sense Media San Francisco, CA 2020 www. commonsensemedia.org/sites/default/files/uploads/research/2020_ zero_to_eight_census_final_web.pdf; abgerufen am 26.9.2021

[18] Stockdale LA, Porter CL, Coyne SM et al. Infants' response to a mobile phone modified still-face paradigm: Links to maternal behaviors and beliefs regarding technoference. Infancy 2020; 25 (5): 571-592. doi:10.1111/infa.12342

[19] Sundqvist A, Koch FS, Birberg et al. Growing Up in a Digital World - Digital Media and the Association With the Child's Language Development at Two Years of Age. Front Psychol 2021; 12: 569920. doi:10.3389/fpsyg.2021.569920

[20] Tronick E. Neurobehavioral and Social Emotional Development. New York: W. W. Norton \& Company; 2007

[21] Wacks Y, Weinstein A. Excessive Smartphone Use Is Associated With Health Problems in Adolescents and Young Adults. Front. Psychiatry 2021; 12: 669042. doi:10.3389/fpsyt.2021.669042

[22] Yeo SL, Schaller A, Robb MB et al. Frequency and Duration of Advertising on Popular Child-Directed Channels on a Video-Sharing Platform. JAMA Netw Open 2021; 4(5): e219890. doi:10.1001/jama networkopen

[23] Yuan N, Weeks HM, Ball R et al. How much do parents actually use their smartphones? Pilot study comparing self-report to passive sensing. Pediatr Res 2019; 86 (4): 416-418. doi:10.1038/s41390-019$0452-2$

[24] Zhao F, Egelman S, Weeks HM et al. Data Collection Practices of Mobile Applications Played by Preschool-Aged Children JAMA Pediatr 2020; 174 (12): e203345. doi:10.1001/jamapediatrics.2020.3345

[25] Spitzer M. Öffnet die Schulen. Nervenheilkunde 2021; 40: 296-311

[26] Anonymus. Kinder dürfen nur noch 3 Stunden pro Woche daddeln. BILD 30.8.2021 www.bild.de/politik/ausland/politik-ausland/chinakinder-duerfen-nur-noch-3-stunden-pro-woche-videospiele-zocken-77535236.bild.html. abgerufen am 30.8.2021 